\title{
Annexin A2 Promotes Development of Retinal Neovascularization through PI3K/ AKT Pathway
}

Chen-yue Li

Changhai Hospital

Zi-chang Zhao

Changhai Hospital

Shi-hong Zhao ( $\square$ zhaosh@smmu.edu.cn )

https://orcid.org/0000-0001-7757-8386

Original investigation

Keywords: AnnexinA2, human retinal endothelial cell, retinal neovascularization, AKT, PI3K, phosphorylation

Posted Date: February 14th, 2020

DOI: https://doi.org/10.21203/rs.2.23554/v1

License: (c) (i) This work is licensed under a Creative Commons Attribution 4.0 International License. Read Full License 
4 Chen-Yue Li, Zi-Chang Zhao, Shi-Hong Zhao*

5 Address: Department of Ophthalmology, Changhai Hospital, The Navy Medical

6 University (The Second Military Medical University), China.

7 E-mail: Chen-Yue Li - 794471016@qq.com; Zi-Chang Zhao -

8 15821693392@163.com; Shi-Hong Zhao*-zhaosh@smmu.edu.cn.

$9 *$ Corresponding author 
13 Chen-Yue Li, Zi-Chang Zhao, Shi-Hong Zhao*

\section{Annexin A2 Promotes Development of Retinal Neovascularization through PI3K/ AKT Pathway}

\section{Abstract}

\section{Background}

RNV is a pathological characteristic of PDR and ANXA2 play an important role in

the process of RNV while the mechanism remains unclear. We explore the role and molecular basis of ANXA2 in the formation of RNV and seek for new potential targets for the prevention and treatment of PDR.

\section{Methods}

Lentivirus containing plasmids which can interfere ANXA2 and overexpress ANXA2 were packaged and infected HRECs, dividing HRECs into 4 groups. Moreover, 1ul SC79 solution was added in shA2 group and 1ul LY294002 solution was added into lentiA2 group. Western Blot was used to detect expression of ANXA2 and changes in phosphorylation degree of major proteins in PI3K/AKT signaling pathway in each group of HRECs. HRECs of all groups were used to perform EDu cell proliferation assay, Transwell cell migration assay and Matrix tube formation assay. C57BL/6J mice were randomly divided into 3 groups. Mice of OIR group were injected intraperitoneally with $0.05 \mathrm{ml}$ PBS every day from 7 th to 10 th day while mice of LY294002 treatment group were injected with $0.05 \mathrm{ml}$ LY294002 solution and no 
31 treatment in the control group. Lectin GS-IB4 fluorescence staining was used to observe

32 RNV in mice in all groups. The expression of ANXA2 in mouse retinas was detected

33 by Westen-blot.

\section{Results}

35 The proliferation, immigration and angiogenesis ability of HRECs is lower in shA2

36 group than shNC and SC79 treatment group while higher in lentiA2 group than lenti-

37 EGFP and LY294002 treatment group. ANXA2 expression is significantly higher in

38 retina of mice in OIR group and LY294002 treatment group. RNV is significantly less

39 severe in LY294002 treatment group than that in OIR group.

\section{Conclusions}

41 ANXA2 can promote development of RNV through PI3K/ AKT Pathway.

42 Keywords: AnnexinA2, human retinal endothelial cell, retinal neovascularization, AKT,

43 PI3K, phosphorylation

\section{Background}

45 Retinal neovascularization $(\mathrm{RNV})$ is a pathological characteristic of proliferative

46 diabetic retinopathy (PDR) ${ }^{[1,2]}$. New vessels invade into the vitreous and neural retinal

47 layers $^{[3]}$. Through retinal vascular leakage, lead to retinal edema, and fibrosis,

48 ultimately causing visual impairment and blindness ${ }^{[4]}$.

49 Annexin A2 (ANXA2), a $39 \mathrm{kDa}$ member of the annexin family of $\mathrm{Ca}^{2+}$ - dependent,

50 phospholipid-binding proteins, is expressed in the majority of cells and tissues and

51 binds to numerous ligands ${ }^{[5,6]}$. ANXA2 is a pleiotropic protein and involved in diverse 
cellular processes such as cell proliferation, differentiation, cell motility and also plays

53 an important role in cytoskeletal organization, exocytosis, endocytosis, invasion,

54 metastasis, fibrinolysis, ion channel formation and so on ${ }^{[5-7]}$.

55 Numerous papers have reported that upregulation of extracellular localization of 56 ANXA2 has a profound impact on invasive and metastatic phenotype of cells and is 57 directly correlated with neovascularization in cancer. ANXA2 can be phosphorylated 58 and subsequent bind to S100A10, which acts as a premise for the localization of its 59 translocation to the cell surface ${ }^{[8,9]}$. As a cell surface receptor for plasminogen $(\mathrm{Plg})$ 60 and tissue plasminogen activator, the ANXA2 complex can prominently accelerate the 61 conversion of Plg to plasmin. As a result, a cascade of matrix metalloproteinases 62 including MMP2 and MMP9 can be activated ${ }^{[10]}$ and ultimately lead to the degradation 63 of fibrin, fibronectin, laminin, and several kinds of collagen ${ }^{[11]}$. The degradation result 64 in the liberation of not only vascular endothelial cells but also pro-angiogenic growth 65 factors which is critical in the initiation of neovascularization ${ }^{[12]}$. Playing a role in the 66 process of neovascularization, ANXA2 may be regarded as a effective target for the 67 development of neovascularization.

68 It has been well studied that PI3K/AKT signaling pathway regulates a wide variety of 69 cellular processes such as cell proliferation, differentiation, survival, transformation 70 and metastasis ${ }^{[13]}$. The pathway also promotes neovascularization and recruitment of 71 inflammatory cells ${ }^{[14]}$.

72 In this study, we assumed that ANXA2 played a role in the process of RNV and used 
73 human retinal endothelial cells (HRECs) aiming at investigating the role and underlying

74 mechanism of ANXA2 in the development of RNV and demonstrating that ANXA2

75 might regulate the formation of RNV through PI3K/AKT pathway.

76 Materials and methods

$77 \quad$ Animals

78 Wild type C57BL /6J mice were purchased from the animal experiment center of the

79 Navy Medical University and housed at room temperature. We used the animals

80 according to the ARVO Statement for the Use of Animals in Ophthalmic and Vision

81 Research.

82 Materials

83 DMEM, FBS, Lipofectamine 2000 and Opti were purchased from Gibco. ECM was

84 purchased from Sciencell. GS-IB4 and Antibodies against glyceraldehyde-3-phosphate

85 dehydrogenase (GAPDH), ANXA2, AKT, p-AKT(Ser473), phosphatidylinositol 3-

86 Kinase (PI3K), p-PI3K(Tyr458), mTOR, p-mTOR(Ser2448), p70 S6 Kinase, p-p70 S6

87 Kinase(Thr389), 4E-BP1, p-4E-BP1(Thr37/46), Tsc2, p-Tsc2(Thr1462), LY294002,

88 SC79 were purchased from Cell Signaling Technology. Gt anti-Rb IgG $(\mathrm{H}+\mathrm{L})$

89 Secondary Antibody was purchased from Invitrogen. Plasmids MDL, REV, VSVG,

90 shNC, shA2, lenti-EGFP, lentiA2 were kindly provided by Microbiology office of The

91 Navy Medical University. Transwell chambers were purchased from Coring Costar.

92 Matrigel was purchased from BD biosciences.

93 Cell lines 
95 cells were purchased from American Type Culture Collection (ATCC) and cultured

96 according to ATCC recommendations. 293T cells were cultured in DMEM and HRECs

97 were cultured in ECM at $37^{\circ} \mathrm{C}$ in a humidified atmosphere of 5\% $\mathrm{CO} 2$.

\section{Lentivirus Packaging and Production}

$99293 \mathrm{~T}$ cells were seeded in the 6-well culture plate in $2 \mathrm{ml}$ of DMEM then incubated at

$10037{ }^{\circ} \mathrm{C}$ with 5\% CO2 and DMEM was replaced by Opti when 293T grew to $80 \%$

101 confluent. We mixed 40ul Lip2000, 3.2ml Opti, 1.554ug MDL, 1.166ug VSVG, 1.166

102 REV then added 3 ug shNC, shA2, lenti-EGFP, lentiA2 respectively into the mixture.

103 Four kinds of mixture were added into different holes and cells were placed at $37{ }^{\circ} \mathrm{C}$

104 with 5\% CO2 immediately. Transfection medium was replaced with 2ml DMEM after

1056 hours of transfection and cells were incubated at $37{ }^{\circ} \mathrm{C}$ with $5 \% \mathrm{CO} 2$ for additional

10648 hours. Finally, lentiviral supernatant was collected and filtered to remove cellular

107 debris.

108 HREC Infection

109 HRECs were seeded in the 24-well culture plate in $2 \mathrm{ml}$ of ECM then incubated at $37^{\circ} \mathrm{C}$

110 with 5\% CO2.1.5ul lentivirus carrying lentiviral vectors shNC, shA2, lenti-EGFP,

111 lentiA2 was respectively added into four different wells when HRECs grew to $30 \%$

112 confluent making HRECs into four groups: shNC, shA2, lenti-EGFP, lentiA2 group.

113 Cells were placed at $37^{\circ} \mathrm{C}$ with $5 \% \mathrm{CO} 2$ immediately and then collected when they

114 grew to $90 \%$ confluent. 


\section{Western Blot}

116 Total protein was extracted by RIPA lysis buffer and separated by sodium dodecyl

117 sulfate-polyacrylamide gel electrophoresis and then transferred onto PVDF membranes.

118 Membranes were blocked with 5\% skim milk at room temperature for $2.5 \mathrm{~h}$ then

119 incubated overnight at $4^{\circ} \mathrm{C}$ with primary antibody against GAPDH (1:1000), AnnxA2

120 (1:1000), AKT(1:1000), p-AKT(Ser473) (1:1000), PI3K(1:1000), p-PI3K(Tyr458)

121 (1:1000), mTOR(1:1000), p-mTOR (Ser2448) (1:1000), p70 S6 Kinase(1:1000), p-p70

122 S6 Kinase(Thr389) (1:1000), 4E-BP1(1:1000), p-4E-BP1 (Thr37/46) (1:1000),

123 Tsc2(1:1000), p-Tsc2(Thr1462) (1:1000). PVDF membranes were incubated with the

124 corresponding secondary antibody at $37{ }^{\circ} \mathrm{C}$ for $2.5 \mathrm{~h}$. Protein bands were finallt

125 visualized by imaging system.

126 EDu Proliferation Assay

$1273.0 \times 10^{4}$ HRECs were seeded in the 96-well culture plate in 200 ul of ECM then

128 incubated at $37{ }^{\circ} \mathrm{C}$ with $5 \% \mathrm{CO}$. Cell nucleuses of all cells and cells in proliferative

129 stage were stained according to the protocol of EDu Kits and then counted under a

130 microscope.

131 Cell Migration Assay

132 Transwell assay was performed to assess the migration and invasive ability of HRECs.

$133100 \mu \mathrm{l}$ cell suspension containing $2.0 \times 10^{4}$ cells and ECM with $0.5 \%$ serum was added

134 into the upper chamber. Lower chamber was filled with $800 \mu \mathrm{ECM}$ with $1 \%$ serum.

135 Cells were cultured at $37{ }^{\circ} \mathrm{C}$ with $5 \% \mathrm{CO} 2$ for next 12 hours and non-migrate ones were 
gently removed with cotton swabs while the cells on the reverse surface of the membrane were fixed with $4 \%$ paraformaldehyde for 20 min and then stained with $0.5 \%$ crystal violet for 2-4h. Number of cells were finally counted under microscope.

\section{Tube Formation Assay}

140 Each well of the 96-well plates was coated with 60-70ul Matrigel. 2.0×104 HRECs were

141 seeded onto the layer of Matrigel and then cultured at $37{ }^{\circ} \mathrm{C}$ with $5 \% \mathrm{CO} 2$ for $2-4 \mathrm{~h}$.

142 Status of tube formation was observed under microscope and the ability of HRECs to

143 form tubular structures was assessed.

144 Mice Model of Oxygen Induced Retinopathy and Treatment of LY294002

14596 mice were randomly divided into normoxia group, oxygen-induced retinopathy (OIR)

146 group and LY294002 treatment group (n=32/group). Mice of the normoxia group were

147 raised in the normal air and mice in the OIR-control group were put in $75 \% \pm 2 \%$ oxygen

148 from P7 to P12 and returned to normal air on P12 in order to produce OIR model and

149 injected intraperitoneally with $0.05 \mathrm{~mL}$ of phosphate-buffered solution (PBS) once a

150 day from P7 to P10. While those of LY294002 treatment group were raised under the

151 same phenomenon with OIR model but then injected intraperitoneally with $0.05 \mathrm{~mL}$ of

152 LY294002 but not PBS once a day from P7 to P10. Three groups of mice were killed

153 on P17. The retinas of left eyes of mice were stained with diluted GS-IB4 while those

154 of the right eyes were used for protein extraction.

\section{Quantitative Assessment of Retinal Neovascularization by GS-IB4 Staining}

156 Retinas were stained with diluted GS-IB4 in room temperature for overnight and then 
157 cut in four radial incisions with the optic disk as center under the microscope. The

158 process of neovascularization was assessed using fluorescence microscope.

\section{Statistical Analysis}

160 As specified in figure legends, group of data are presented as mean \pm SD error and were

161 analyzed using t-test. Asterisks in each box and whisker plot indicate statistical

162 significance $(*=\mathrm{P}<0.05 ; * *=\mathrm{P}<0.01 ; * * *=\mathrm{P}<0.001)$.

\section{Results}

164 ANXA2 is closely related to PI3K/AKT signaling pathway in HRECs

165 We infected HRECs with lentivirus carrying lentiviral vectors shNC, shA2, lenti-EGFP,

166 lentiA2 and made HRECs in four groups. Moreover, we also added AKT activator

167 SC79 in shA2 group and PI3K inhibitor LY294002 in lentiA2 group. Western blot was

168 performed to verify the relationship between ANXA2 and PI3K/AKT signaling

169 pathway in HRECs. ShA2 group showed the degradation of phosphorylation of AKT

170 and related proteins in the pathway and the best silencing efficiency on the PI3K/AKT

171 signaling pathway while the changes were counteracted in the shA2+SC79 group.

172 However, the level of phosphorylation of AKT and related proteins in the pathway was

173 obviously enhanced in lentiA2 group and the up-regulation was dampened when

174 LY294002 was added (Figure 1A, 1B, 1C, 1D, 1E). 


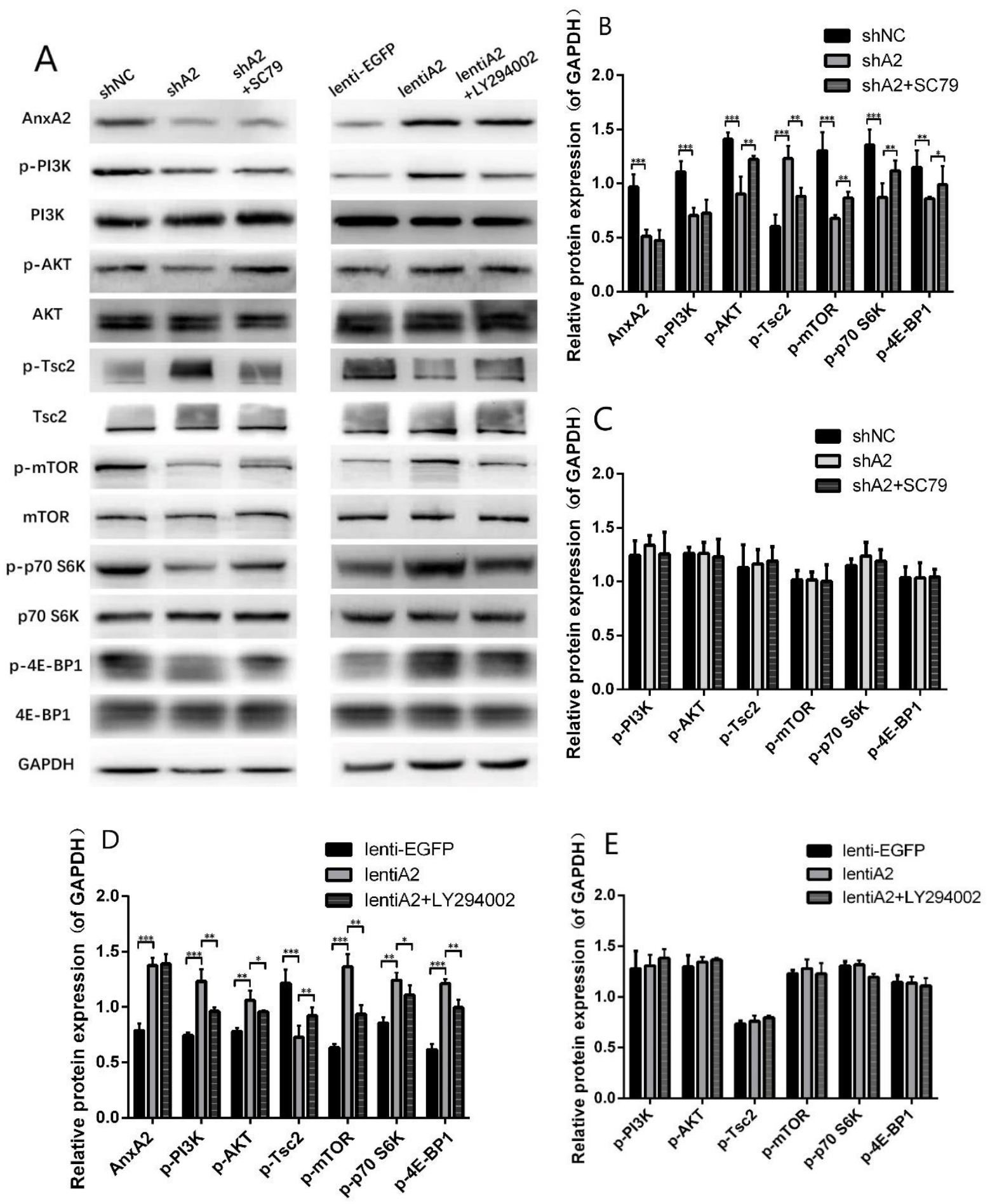

177 Figure 1 The interference and overexpression of ANXA2 obviously influence the

178 phosphorylation levels of important proteins in PI3K/ AKT signaling pathway in HRECs cells

179 of each group. Western blot assay for protein expression of AXNA2, p-PI3K, PI3K, p-AKT, AKT, 
181 (A); Statistical analysis (B, C, D, E) * $\mathrm{P}<0.05, * * \mathrm{P}<0.01$, *** $\mathrm{P}<0.001, \mathrm{n}=3$.

182 ANXA2 promotes proliferation of HRECs through PI3K/AKT signaling pathway

183 EDu assay was performed to investigate whether ANXA2 can influence the

184 proliferation on HRECs. The result shows number of HRECs in proliferative stage in

185 lentiA2 group was larger than that in lenti-EGFP group but the number decreased

186 when LY294002 was added. While the number of HRECs in proliferative stage in shA2

187 group was smaller compared to shNC group. However, proliferation of HRECs in shA2

188 group was strengthen when SC79 was Added. These results indicated that ANXA2

189 could promote proliferation of HRECs through PI3K/AKT signaling pathway (Figure

$1902 \mathrm{~A}$ and 2B).

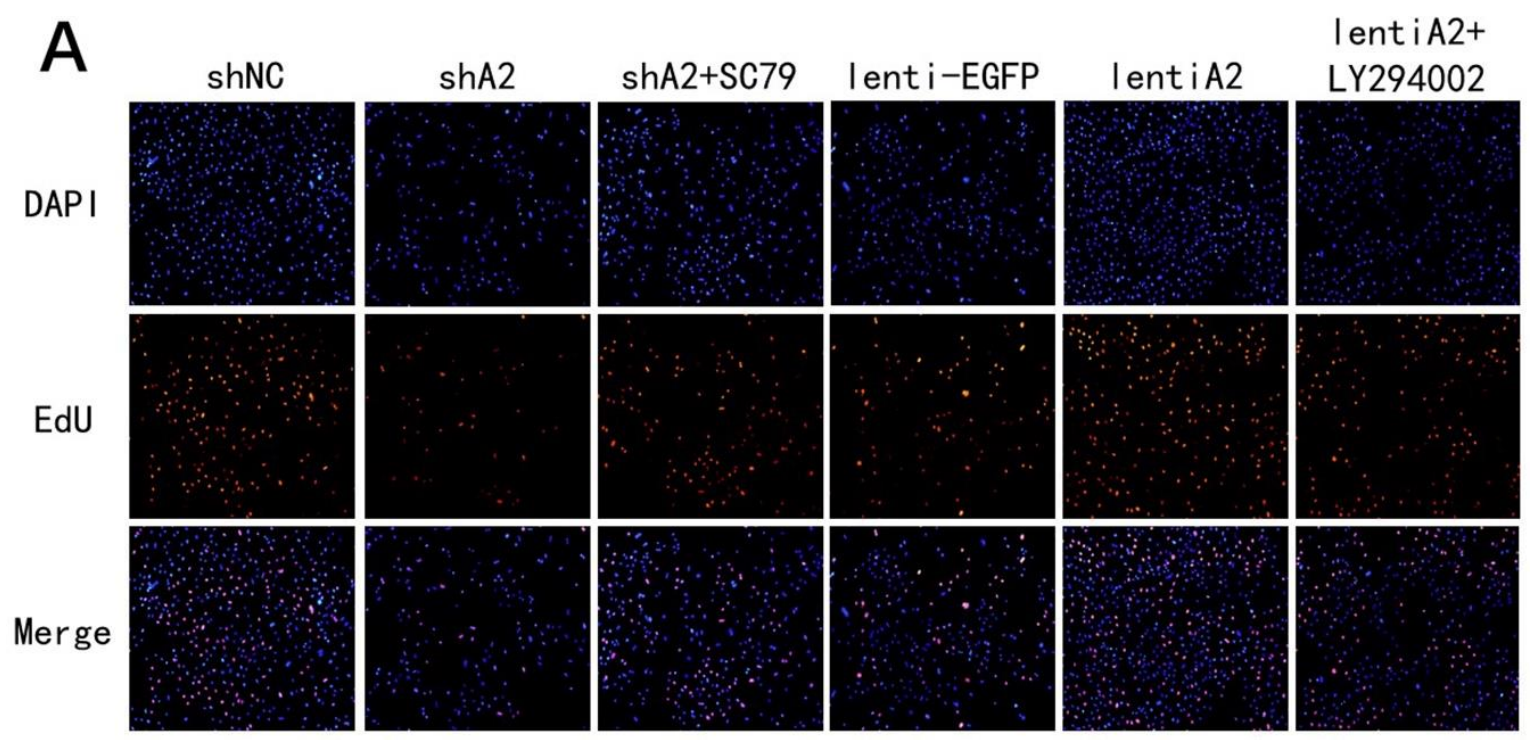



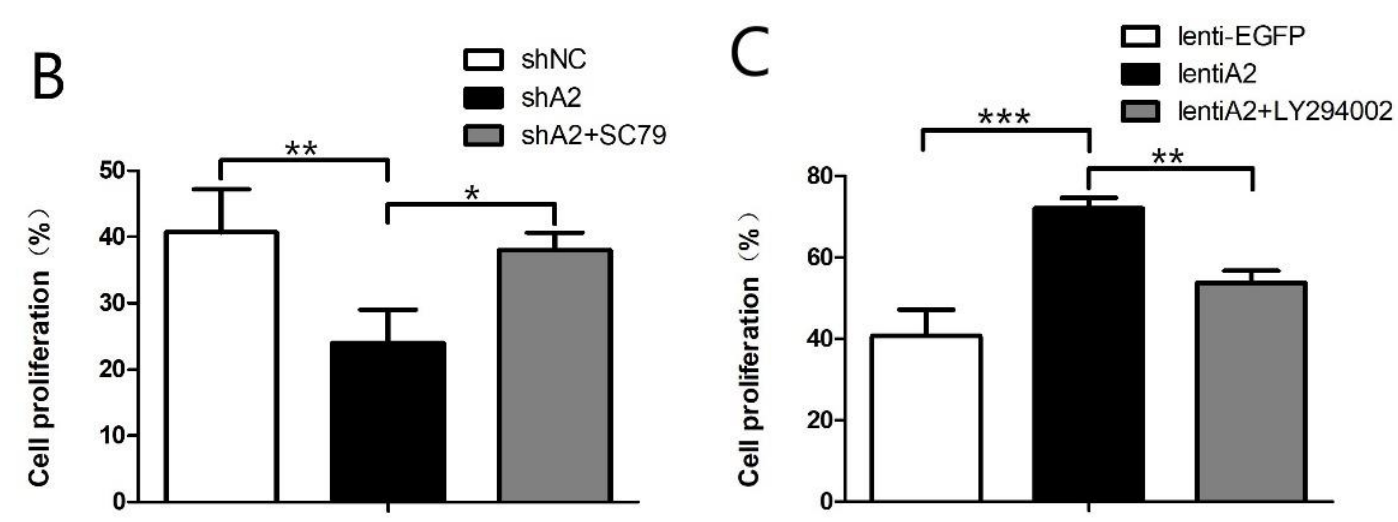

192

193 Figure 2 The interference and overexpression of ANXA2 obviously influence the

194 proliferation of HRECs and the influence is highly related with PI3K/AKT signaling

195 pathway. EDu assay show the proliferation of HRECs in each group(A). Statistical analysis (B, C)

$196 * * \mathrm{P}<0.01, * * * \mathrm{P}<0.001, \mathrm{n}=3$.

197 ANXA2 promotes migration and invasion of HRECs through PI3K/AKT signaling

198 pathway

199 To explore the influence of ANXA2 in HRECs, Transwell assay was performed and

200 indicated that ANXA2 could significantly enhance the ability of migration and invasion

201 of HRECs in lentiA2 group while the impact was counteracted by LY294002. On the

202 contrary, HRECs in shA2 showed low migration and invasion but the ability was

203 restored by SC79, the result confirmed that ANXA2 may promote migration and

204 invasion of HRECs through PI3K/AKT signaling pathway (Figure 3A and 3B). 
A

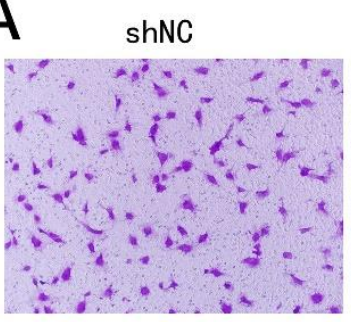

lent i-EGFP

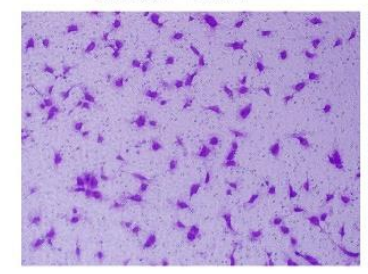

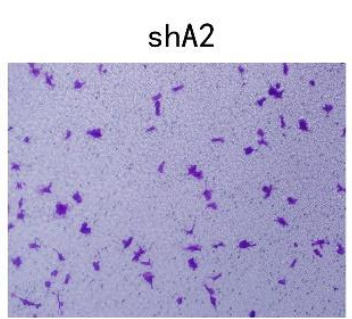

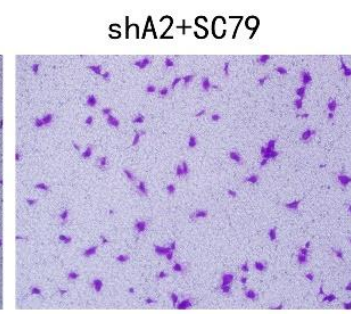

lentiA2+

LY294002
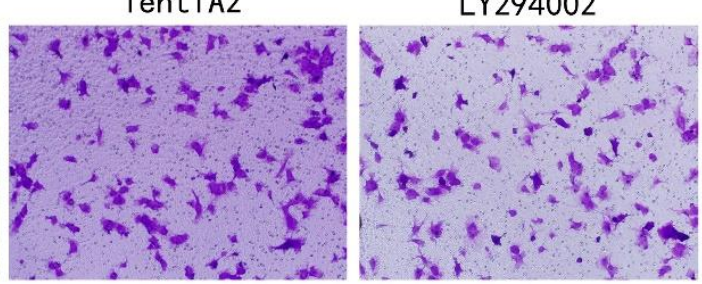
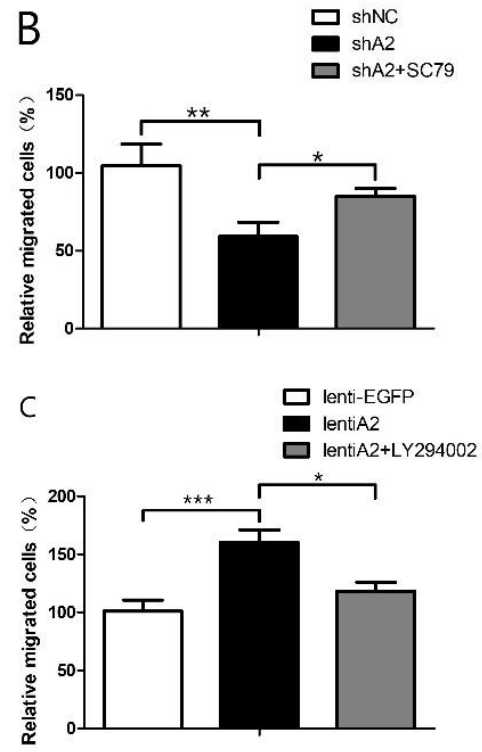

Figure 3 The interference and overexpression of ANXA2 obviously influence the migration and invasion of HRECs and the influence is highly related with PI3K/AKT signaling pathway.

Transwell assay show the migration and invasion of HRECs in each group(A). Statistical analysis $(\mathrm{B}, \mathrm{C}) * \mathrm{P}<0.05, \quad * * \mathrm{P}<0.01, \quad * * * \mathrm{P}<0.001, \mathrm{n}=3$ 。

213 We performed tube formation assay to explore the potential relationship between

214 ANXA2 and angiogenesis which plays a critical role in RNV. Tube formation assay

215 revealed that HRECs in lentiA2 group had better angiogenesis compared with lenti-

216 EGFP group while the angiogenesis ability of cells was dampened when LY294002 was

217 added. While the HRECs in shA2 group had poorer angiogenesis compared to shNC

218 group. However, the ability of HRECs in shA2 group was restored when SC79 was

219 Added. These results indicated that ANXA2 could promote the expression of RNV and 
220 facilitate angiogenesis ability of HRECs and the influence might be generated through

221 PI3K/AKT signaling pathway (Figure 4A and 4B).

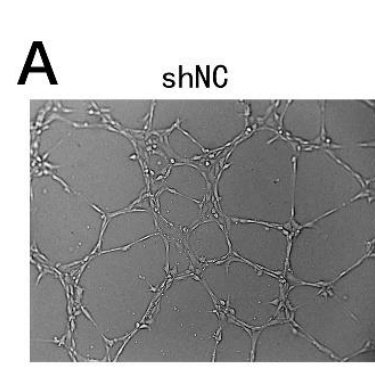

lent i-EGFP

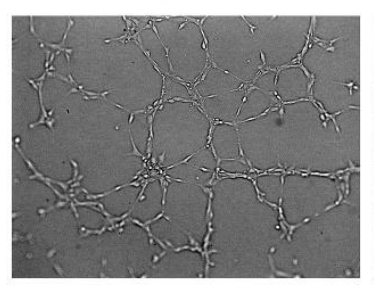

223
shA2

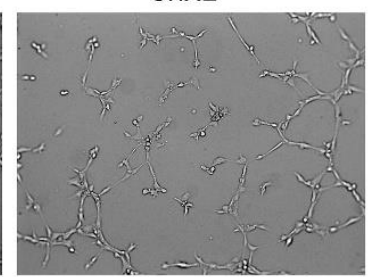

lentiA2

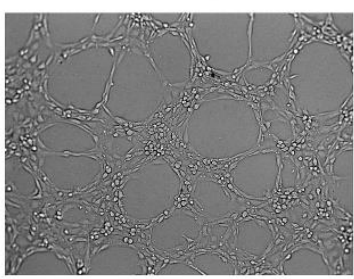

$\operatorname{shA} 2+\operatorname{SC} 79$

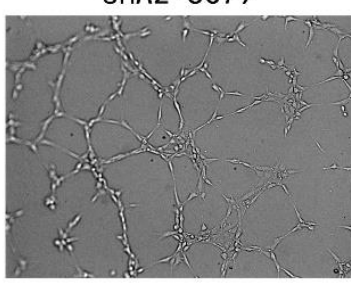

lent iA2+

LY294002

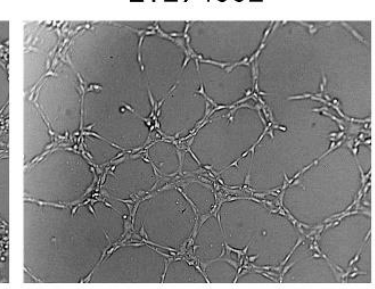

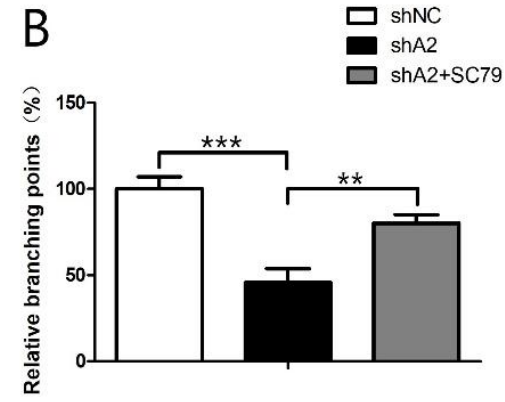

C

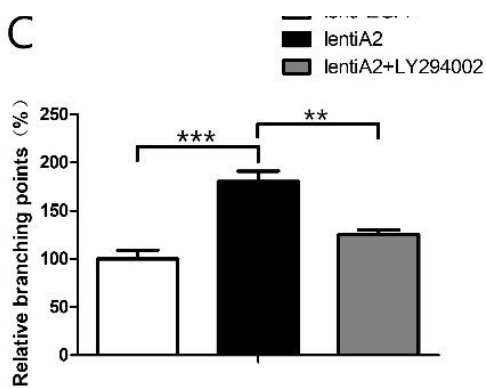

224 Figure 4 The interference and overexpression of ANXA2 obviously influence the angiogenesis

225 ability of HRECs and the influence is highly related with PI3K/AKT signaling pathway. Tube

226 formation assay show the angiogenesis ability of HRECs in each group(A). Statistical analysis

$(\mathrm{B}, \mathrm{C}) * * \mathrm{P}<0.01, * * * \mathrm{P}<0.001, \mathrm{n}=3$ 。

228 Expression of ANXA2 is significantly increased in the retina of mice of OIR model

229 and the inhibition of PI3K/AKT signaling pathway results in receding the process

230 of neovascularization

231 Western-blot was applied to measure the expression level of ANXA2 in the mice of

232 normoxia, OIR and LY294002 treatment group. ANXA2 expression is significantly

233 higher in the other two groups than normoxia group and that indicated high level of

234 expression of ANXA2 is closely related to the development of retinal 
235 neovascularization (Figure 5). Retinal vessels of the mice in normoxia group stretched 236 out from the optic disc and distributed radially and we could hardly see the non237 perfusion area. The diameter of vessels was thick and there were no abnormal branches.

238 On the contrast, the retina of the OIR group showed a large amount of non-perfusion 239 area in the center which was surrounded by bright and disorderly neovascular plexus.

240 While the neovascular plexus, nonperfusion and abnormal branches were much less 241 severe in the retina of mice in LY294002 treatment group (Figure 6).
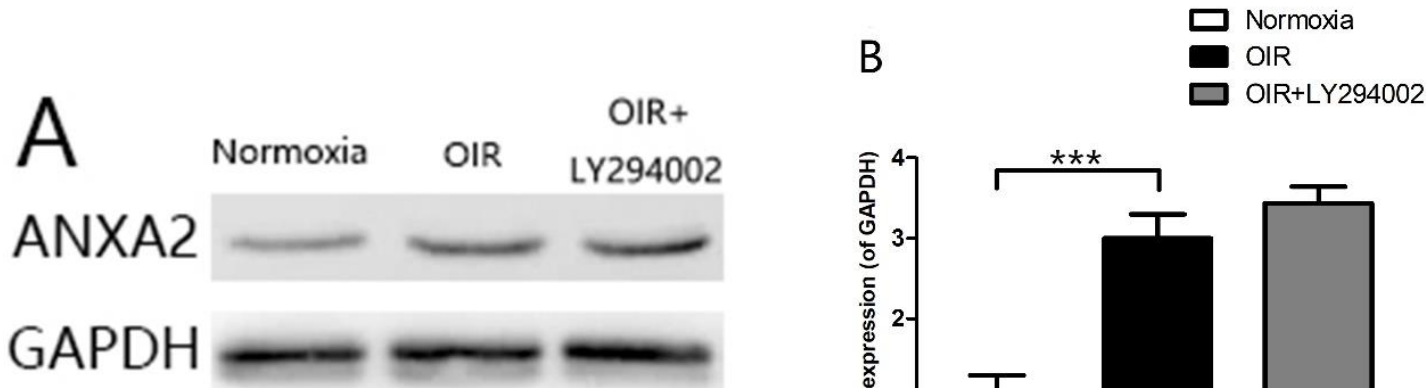

245 Figure 5 ANXA2 expression is significantly higher in retina of mice in OIR group and

246 LY294002 treatment group. Western blot assay for protein expression of AXNA2 in retina of mice

247 of each group (A); Statistical analysis (B) $* * * \mathrm{P}<0.001, \mathrm{n}=26$. 

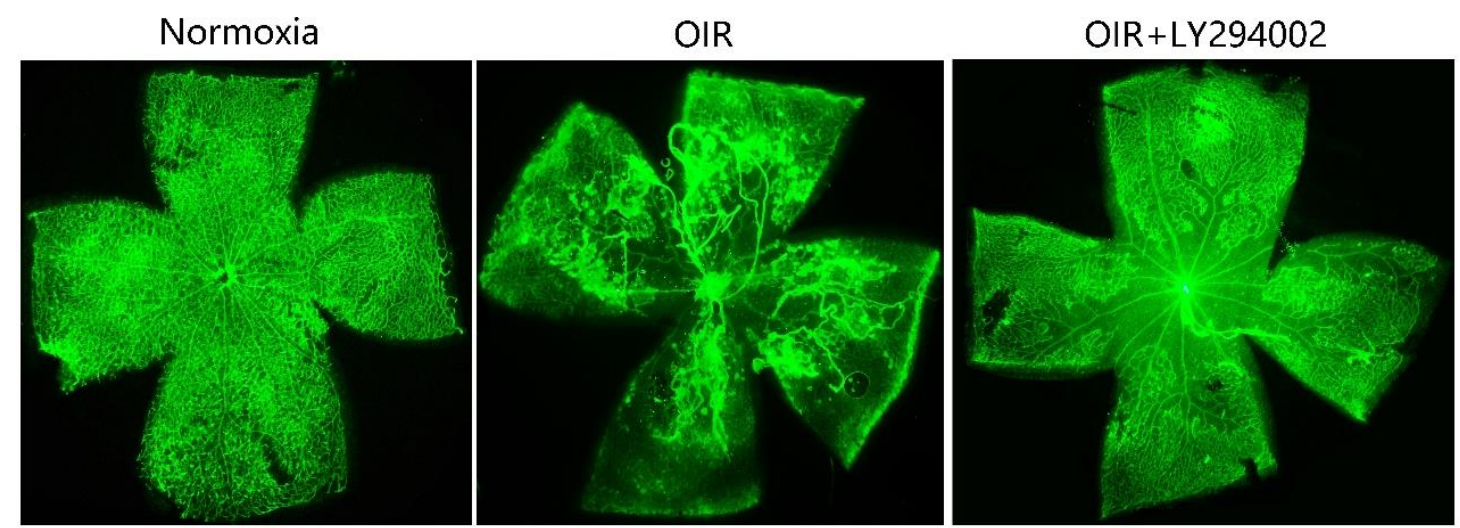

Figure 6 Inhibition of PI3K/AKT signaling pathway significantly recede the process of

neovascularization. Images are typical retinal angiogram of the retina of mice in the normoxia

251 group, OIR group and LY294002 treatment group. $\mathrm{n}=26$.

\section{Discussion}

253 ANXA2 is a kind of $\mathrm{Ca}^{2+}$ - dependent protein which can bind to phospholipid. Previous 254 studies pointed out a variety of biological functions of ANXA2 such as participating in 255 cell signal transduction, cell cytoskeletal movement, cell proliferation, apoptosis and 256 neovascularization ${ }^{[15]}$. Multiple pieces of evidence suggested that ANXA2 is highly 257 expressed in the vascular system and overexpressed in angiogenesis, suggesting that it 258 may play an important role in angiogenesis ${ }^{[16]}$. Immunohistochemical studies 259 of human breast tumor tissues provided compelling evidence of a strong positive 260 correlation between ANXA2 expression and neovascularization and suggested that 261 ANXA2 was a potential target to delay or inhibit breast tumor growth by 262 inhibiting neovascularization ${ }^{[17]}$. The addition of an antibody against ANXA2 also 263 produced a prominent inhibition of network formation of human umbilical vein 264 endothelial cells in a co-culture system mimicking the process of neovascularization 
${ }^{[18]}$. However, the accurate function of ANXA2 in PDR has not been illustrated clearly.

266 PI3K/AKT signaling pathway regulates a wide variety of cellular processes, such as

267 cell proliferation, differentiation, survival, cell transformation and metastasis of cells ${ }^{[19]}$.

268 It has been well documented that under the stimulation of Vascular endothelial growth

269 factor (VEGF), AKT could promote proliferation and migration of vascular endothelial

270 cell and participated in the process of vascular remodeling ${ }^{[20]}$. The lack of AKT can

271 intensify the process of atherosclerosis in the mice model ${ }^{[21]}$. The PI3K/AKT pathway

272 has close relationship with tumor progression and abnormity of this pathway can be

273 frequently observed in a variety of tumors ${ }^{[22-24]}$. In both rabbit cornea model ${ }^{[25]}$ and

274 mouse ischemic retinal model ${ }^{[26]}$, PI3K/AKT pathway were confirmed to contain

275 effective angiogenesis characteristics. Moreover, it was found the level of

276 phosphorylation of AKT was significantly reduced while the total amount of AKT

277 remains when the gastric cancer cells were transfected with small interfering RNA

278 containing shA2 plasmid which further indicated that ANXA2 may play a role by

279 influencing the phosphorylation of AKT ${ }^{[27]}$. When ANXA2 expression were inhibited,

280 the levels of phosphorylation of AKT and PI3K, the key moleculars of AKT pathway,

281 were distinctly lower and the same phenomenon also appeared when PI3K inhibitors

282 were added in cells ${ }^{[28]}$. The result suggested ANXA2 might accelerate cell proliferation

283 and migration by promoting the phosphorylation of AKT and related proteins in the

284 pathway ${ }^{[28]}$.

285 Neovascularization, a complex process for new blood vessels formation in which 
endothelial cells migration, adhesion and tubular formation play a very important role

$287{ }^{[29]}$, is a pathological hallmark feature for PDR ${ }^{[30]}$. Edu assay, Transwell cell migration 288 assay and tube formation assay are mature methods to simulate in vitro 289 neovascularization ${ }^{[31-33]}$. We found that ANXA2 can promote the proliferation, 290 migration and tube formation of HRECs. The results indicated that ANXA2 can be a 291 positive regulator of human retinal neovascularization, which is consistent with the role 292 of ANXA2 in endothelial cells ${ }^{[34]}$ while the impact could be significantly weakened by 293 the PI3K/AKT pathway inhibitor. These phenotypes had proved our hypothesis that 294 ANXA2 promotes development of retinal neovascularization through PI3K/ AKT 295 pathway.

296 To investigate its role in retinal neovascularization, we also constructed OIR mice 297 model, the most mature and classic model of retinal neovascularization ${ }^{[35]}$. To ascertain 298 whether we built the model effectively, we selected the P17 mice in all three groups 299 because P17 is the peak of the formation of retinal neovascularization in OIR model 300 and new blood vessels then begin to fade and is almost completely replaced by normal 301 vascular system on P25 ${ }^{[36]}$. Compared with STZ-induced diabetic models, OIR mice 302 model reveals not only the process of vaso-obliteration but also neovascularization 303 phase ${ }^{[37]}$, which is consistent with PDR and inspired our particularly strong focus. 304 Therefore, we employed OIR mice model to illustrate the role of ANXA2 in PDR. Then 305 we measured the protein expression level of ANXA2 in mice retinal, and the results 306 showed the expression of ANXA2 in the OIR group was significantly increased in 
308 of retinal neovascularization in mice and play an important role in retinal

309 neovascularization ${ }^{[16]}$.

310 Furthermore, we hypothesized that the development of retinal neovascularization is

311 highly related to PI3K/AKT pathway and injected $0.05 \mathrm{ml}$ PI3K inhibitor LY294002

312 intraperitoneally once a day from P7 to P10. Finally, we found the retinas of mice which

313 was treated with LY294002 contained less severe neovascular and irregular expansion.

314 VEGF proved a key factor of hypoxia-inducible RNV and also an effective target to

315 inhibit the progress of it. What's more, intravitreal injection of anti-VEGF medicine

316 has led a significant breakthrough in the treatment of RNV ${ }^{[38,39]}$. Inhibitors of VEGF

317 show great promise for treatment of RNV. However, a growing number of researches

318 suggest that the strategy may not address the underlying causes of pathology and have

319 some noteworthy deleterious side-effects including the acceleration of the formation of

320 proliferative epi-retinal membranes and aggravation of vitreoretinal traction ${ }^{[40]}$.

321 Increased incidence of systemic adverse events had scattered across many organ

322 systems induced by anti-VEGF treatment, the significance of which remains unclear

$32{ }^{[41]}$. In addition, the effect of therapy can just last for about several months which means

324 the patients have to suffer repeat intravitreal injection of the medicine. Therefore, the

325 risk of intravitreal infection is much higher and some patients also respond poorly to

326 anti-VEGF treatments ${ }^{[42]}$.Thus, identification of novel targets that play important roles

327 in retinal neovascularization is urgently needed for those not responsible for anti-VEGF 
328 therapy ${ }^{[43]}$. Studies also demonstrated that celecoxib could inhibit the expression of

329 VEGF and neovascularization growth in retinal pigment epithelial cells and the effect

330 was mediated through a PI3K/AKT-dependent manner ${ }^{[44]}$ and abrogating of VEGF

331 significantly reduced the risk of developing to PDR in type 2 diabetes patients ${ }^{[45]}$.

\section{Conclusions}

333 ANXA2 can promote development of RNV through PI3K/ AKT Pathway. We can

334 make ANXA2 and PI3K/ AKT pathway as a potential target for the regulation of

335 pathological neovascularization of retina, which also provides a novel idea for effective

336 prevention and treatment of PDR in the future.

$337 \quad$ Availability of data and material

338 All data generated or analysed during this study are included in this published article.

339 Abbreviations

$340 \quad$ ANXA2: Annexin A2

341 ATCC; American Type Culture Collection

342 GAPDH: glyceraldehyde-3-phosphate dehydrogenase

343 HRECs: human retinal endothelial cells

344 OIR: oxygen-induced retinopathy

345 PDR: proliferative diabetic retinopathy

346 Plg: plasminogen

347 RNV: Retinal neovascularization

348 Ethics declarations 
350 The experimental protocol was established, according to the ethical guidelines of the

351 Helsinki Declaration. Written informed consent was obtained from individual or

352 guardian participants.

\section{Consent for publication}

354 All authors read and approved the final version of manuscript.

\section{Competing interests}

356 The authors declare that they have no competing interests.

\section{Author information}

\section{Affiliations}

359 Department of Ophthalmology, Changhai Hospital, The Navy Medical University (The

360 Second Military Medical University), China.

\section{Contributions}

362 Chen-Yue Li and Shi-Hong Zhao designed the study, Chen-Yue Li performed the tasks

363 of study, Zi-Chang Zhao analyzed the data, Chen-Yue Li wrote the manuscript.

364 Corresponding author

365 Correspondence to zhaosh@smmu.edu.cn.

\section{Funding}

367 This study was supported by grants from National Natural Science Foundation of

368 China, NO.81970822.

\section{Acknowledgements}


materials in the experiment.

372

373

374

375

376

377

378

379

380

381

382

383

384

385

386

387

388

389

390

391

392

393

394

395

396

397

398

399

400

401

402

403

404

405

406

407

408

\section{References}

[1] Wang S, Ji LY, Li L, Li JM. Oxidative stress, autophagy and pyroptosis in the neovascularization of oxygen-induced retinopathy in mice. Mol Med Rep. 2019. 19(2): 927-934.

[2] Zhang W, Li Y, Nguyen VP, et al. High-resolution, in vivo multimodal photoacoustic microscopy, optical coherence tomography, and fluorescence microscopy imaging of rabbit retinal neovascularization. Light Sci Appl. 2018. 7: 103.

[3] Wong TY, Cheung CM, Larsen M, Sharma S, Simó R. Diabetic retinopathy. Nat Rev Dis Primers. 2016. 2: 16012.

[4] Al-Shabrawey M, Elsherbiny M, Nussbaum J, Othman A, Megyerdi S, Tawfik A. Targeting Neovascularization in Ischemic Retinopathy: Recent Advances. Expert Rev Ophthalmol. 2013. 8(3): 267-286.

[5] Gabel M, Delavoie F, Royer C, et al. Phosphorylation cycling of Annexin A2 Tyr23 is critical for calcium-regulated exocytosis in neuroendocrine cells. Biochim Biophys Acta Mol Cell Res. 2019.

[6] Murphy AG, Foley K, Rucki AA, et al. Stromal Annexin A2 expression is predictive of decreased survival in pancreatic cancer. Oncotarget. 2017. 8(63): 106405-106414.

[7] Lokman NA, Ho R, Gunasegaran K, Bonner WM, Oehler MK, Ricciardelli C. Anti-tumour effects of all-trans retinoid acid on serous ovarian cancer. J Exp Clin Cancer Res. 2019. 38(1): 10.

[8] Holthenrich A, Gerke V. Regulation of von-Willebrand Factor Secretion from Endothelial Cells by the Annexin A2-S100A10 Complex. Int J Mol Sci. 2018. 19(6).

[9] He KL, Sui G, Xiong H, et al. Feedback regulation of endothelial cell surface plasmin generation by PKC-dependent phosphorylation of annexin A2. J Biol Chem. 2011. 286(17): 15428-39.

[10] Netzel-Arnett S, Mitola DJ, Yamada SS, et al. Collagen dissolution by keratinocytes requires cell surface plasminogen activation and matrix metalloproteinase activity. J Biol Chem. 2002. 277(47): 45154-61.

[11] Visse R, Nagase H. Matrix metalloproteinases and tissue inhibitors of metalloproteinases: structure, function, and biochemistry. Circ Res. 2003. 92(8): 827-39.

[12] Potente M, Gerhardt H, Carmeliet P. Basic and therapeutic aspects of angiogenesis. Cell. 2011. 146(6): 873-87. 
[13] Méndez-Pertuz M, Martínez P, Blanco-Aparicio C, et al. Modulation of

[14] Hirsch E, Ciraolo E, Franco I, Ghigo A, Martini M. PI3K in cancer-stroma

[15] Yang W, Mei FC, Cheng X. EPAC1 regulates endothelial annexin A2 cell surface translocation and plasminogen activation. FASEB J. 2018. 32(4): 22122222.

[16] Zhao S, Huang L, Wu J, Zhang Y, Pan D, Liu X. Vascular endothelial growth factor upregulates expression of annexin A2 in vitro and in a mouse model of ischemic retinopathy. Mol Vis. 2009. 15: 1231-42.

[17] Sharma M, Blackman MR, Sharma MC. Antibody-directed neutralization of annexin II (ANX II) inhibits neoangiogenesis and human breast tumor growth in a xenograft model. Exp Mol Pathol. 2012. 92(1): 175-84.

[18] Raddum AM, Evensen L, Hollås H, Grindheim AK, Lorens JB, Vedeler A. Domains I and IV of annexin A2 affect the formation and integrity of in vitro capillary-like networks. PLoS One. 2013. 8(3): e60281.

[19] Ouyang ZH, Wang WJ, Yan YG, Wang B, Lv GH. The PI3K/Akt pathway: a critical player in intervertebral disc degeneration. Oncotarget. 2017. 8(34): 57870-57881.

[20] Manning BD, Toker A. AKT/PKB Signaling: Navigating the Network. Cell. 2017. 169(3): 381-405.

[21] Fernández-Hernando C, József L, Jenkins D, Di Lorenzo A, Sessa WC. Absence of Akt1 reduces vascular smooth muscle cell migration and survival and induces features of plaque vulnerability and cardiac dysfunction during atherosclerosis. Arterioscler Thromb Vasc Biol. 2009. 29(12): 2033-40.

[22] Sathe A, Nawroth R. Targeting the PI3K/AKT/mTOR Pathway in Bladder Cancer. Methods Mol Biol. 2018. 1655: 335-350.

[23] Li JF, Li WH, Xue LL, Zhang Y. Long non-coding RNA PICART1 inhibits cell proliferation by regulating the PI3K/AKT and MAPK/ERK signaling pathways in gastric cancer. Eur Rev Med Pharmacol Sci. 2019. 23(2): 588-597.

[24] Fu Q, Huang Y, Ge C, et al. SHIP1 inhibits cell growth, migration, and invasion in non-small cell lung cancer through the PI3K/AKT pathway. Oncol Rep. 2019. 41(4): 2337-2350.

[25] Chen J, Shao C, Lu W, et al. Adenosine triphosphate-induced rabbit corneal endothelial cell proliferation in vitro via the P2Y2-PI3K/Akt signaling axis. Cells Tissues Organs. 2014. 199(2-3): 131-9.

[26] Sharma S, Guru SK, Manda S, et al. A marine sponge alkaloid derivative 4chloro fascaplysin inhibits tumor growth and VEGF mediated angiogenesis by disrupting PI3K/Akt/mTOR signaling cascade. Chem Biol Interact. 2017. 275: 47-60.

[27] Zhang ZD, Li Y, Fan Q, Zhao B, Tan B, Zhao XF. Annexin A2 is implicated in multi-drug-resistance in gastric cancer through p38MAPK and AKT pathway. 
Neoplasma. 2014. 61(6): 627-37.

[28] $\mathrm{Li} \mathrm{Z}, \mathrm{He} \mathrm{T}, \mathrm{Du} \mathrm{K}$, et al. Inhibition of oxygen-induced ischemic retinal neovascularization with adenoviral 15-lipoxygenase-1 gene transfer via upregulation of PPAR $-\gamma$ and down-regulation of VEGFR-2 expression. PLoS One. 2014. 9(1): e85824.

[29] Xu Q, Bai Y, Huang L, Zhou P, Yu W, Zhao M. Knockout of $\alpha$ A-crystallin inhibits ocular neovascularization. Invest Ophthalmol Vis Sci. 2015. 56(2): 81626.

[30] Abu El-Asrar AM, Ahmad A, Alam K, et al. Extracellular matrix metalloproteinase inducer (EMMPRIN) is a potential biomarker of angiogenesis in proliferative diabetic retinopathy. Acta Ophthalmol. 2017. 95(7): 697-704.

[31] Laohavisit A, Davies JM. Annexins. New Phytol. 2011. 189(1): 40-53.

[32] Law EW, Cheung AK, Kashuba VI, et al. Anti-angiogenic and tumorsuppressive roles of candidate tumor-suppressor gene, Fibulin-2, in nasopharyngeal carcinoma. Oncogene. 2012. 31(6): 728-38.

[33] Song H, Pan D, Sun W, et al. SiRNA directed against annexin II receptor inhibits angiogenesis via suppressing MMP2 and MMP9 expression. Cell Physiol Biochem. 2015. 35(3): 875-84.

[34] Luo M, Hajjar KA. Annexin A2 system in human biology: cell surface and beyond. Semin Thromb Hemost. 2013. 39(4): 338-46.

[35] Ishikawa K, Yoshida S, Nakao S, et al. Bone marrow-derived monocyte lineage cells recruited by MIP-1 $\beta$ promote physiological revascularization in mouse model of oxygen-induced retinopathy. Lab Invest. 2012. 92(1): 91-101.

[36] Eresch J, Stumpf M, Koch A, et al. Sphingosine Kinase 2 Modulates Retinal Neovascularization in the Mouse Model of Oxygen-Induced Retinopathy. Invest Ophthalmol Vis Sci. 2018. 59(2): 653-661.

[37] Barcelona PF, Sitaras N, Galan A, et al. p75NTR and Its Ligand ProNGF Activate Paracrine Mechanisms Etiological to the Vascular, Inflammatory, and Neurodegenerative Pathologies of Diabetic Retinopathy. J Neurosci. 2016. 36(34): 8826-41.

[38] Seo S, Suh W. Antiangiogenic effect of dasatinib in murine models of oxygeninduced retinopathy and laser-induced choroidal neovascularization. Mol Vis. 2017. 23: 823-831.

[39] Saravia M, Zeman L, Ingolotti M, Schlaen A. The VEGF paradox: Does diabetic retinopathy protect from age related macular degeneration. Med Hypotheses. 2017. 109: 156-161.

[40] Käll A. Is Avastin the right choice of treatment for retinopathy of prematurity. Acta Paediatr. 2012. 101(8): 796-8.

[41] CATT Research Group, Martin DF, Maguire MG, et al. Ranibizumab and bevacizumab for neovascular age-related macular degeneration. N Engl J Med. 2011. 364(20): 1897-908. 
[42] Wong RK, Hubschman S, Tsui I. Reactivation of retinopathy of prematurity after ranibizumab treatment. Retina. 2015. 35(4): 675-80.

[43] Zhang L, Fu X, Zeng H, et al. Microarray Analysis of Long Non-Coding RNAs and Messenger RNAs in a Mouse Model of Oxygen-Induced Retinopathy. Int J Med Sci. 2019. 16(4): 537-547.

[44] Sun YZ, Cai N, Liu NN. Celecoxib Down-Regulates the Hypoxia-Induced Expression of HIF-1 $\alpha$ and VEGF Through the PI3K/AKT Pathway in Retinal Pigment Epithelial Cells. Cell Physiol Biochem. 2017. 44(4): 1640-1650.

[45] Chiefari E, Ventura V, Capula C, et al. A polymorphism of HMGA1 protects against proliferative diabetic retinopathy by impairing HMGA1-induced VEGFA expression. Sci Rep. 2016. 6: 39429. 
Figures

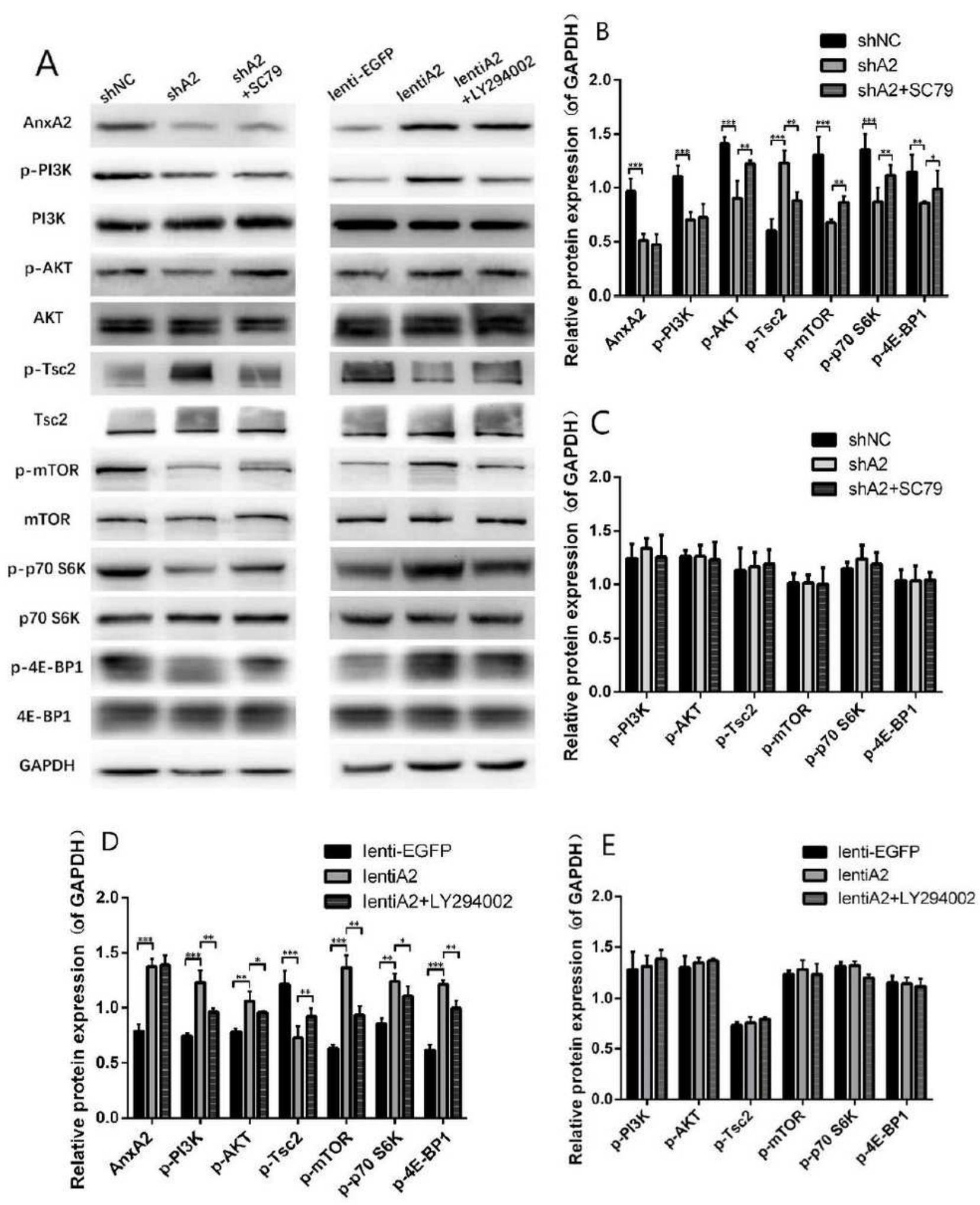

Figure 2

The interference and overexpression of ANXA2 obviously influence the phosphorylation levels of important proteins in PI3K/ AKT signaling pathway in HRECs cells of each group. Western blot assay for protein expression of AXNA2, p-PI3K, PI3K, p-AKT, AKT, p-Tsc2, Tsc2, p-mTOR, mTOR, p-p70 S6K, p70 S6K, 
p-4E-BP1, 4E-BP1 in HRECs of each group (A); Statistical analysis $(B, C, D, E)$ * $P<0.05 \nabla^{\star \star} P<0.01 \nabla^{\star \star \star *}$ $P<0.001 \rrbracket n=3$.
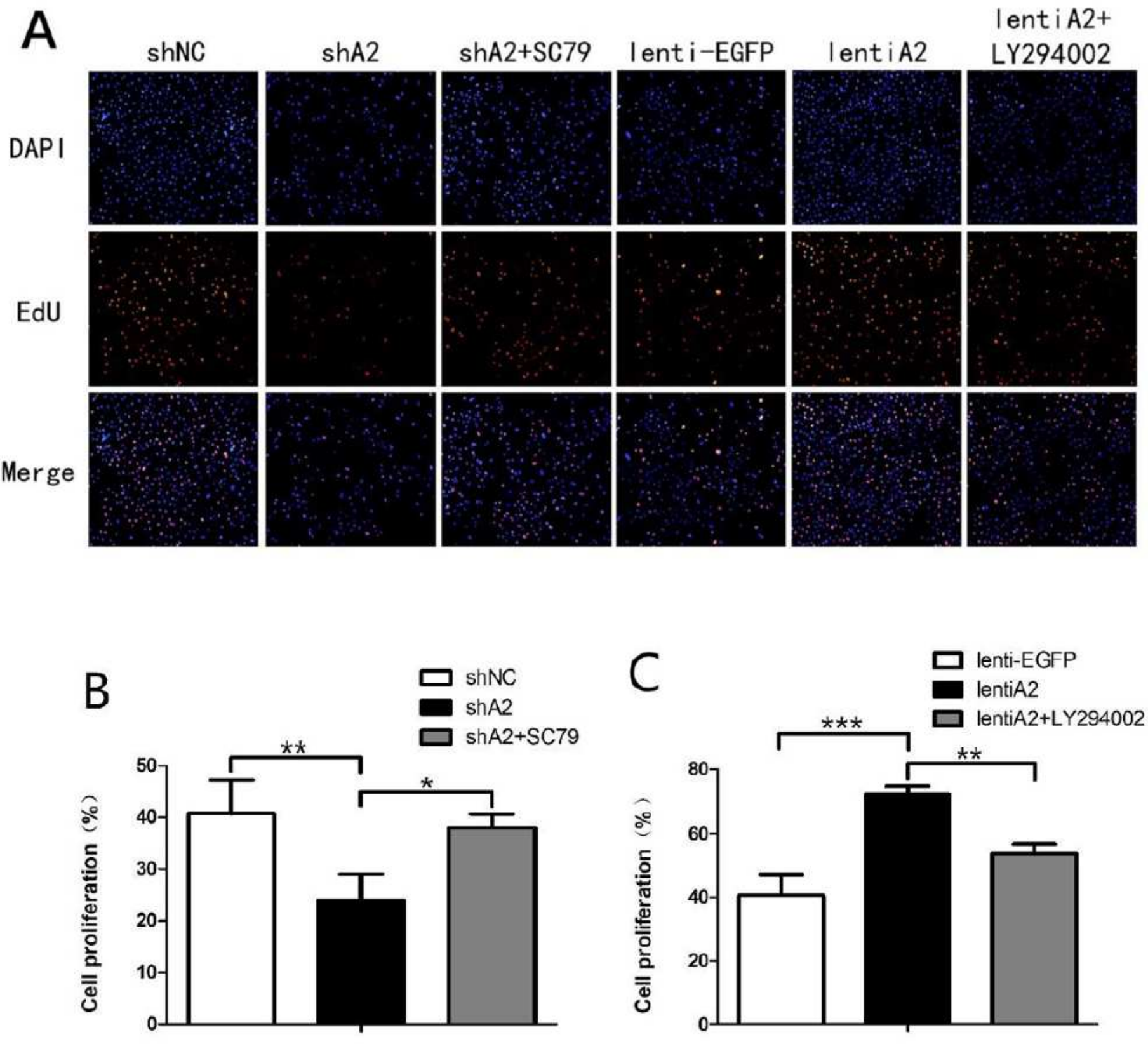

Figure 4

The interference and overexpression of ANXA2 obviously influence the proliferation of HRECs and the influence is highly related with PI3K/AKT signaling pathway. EDu assay show the proliferation of HRECs in each group $(A)$. Statistical analysis $(B, C) * * P<0.01 \rrbracket \star \star \star * P<0.001 \rrbracket n=3$. 

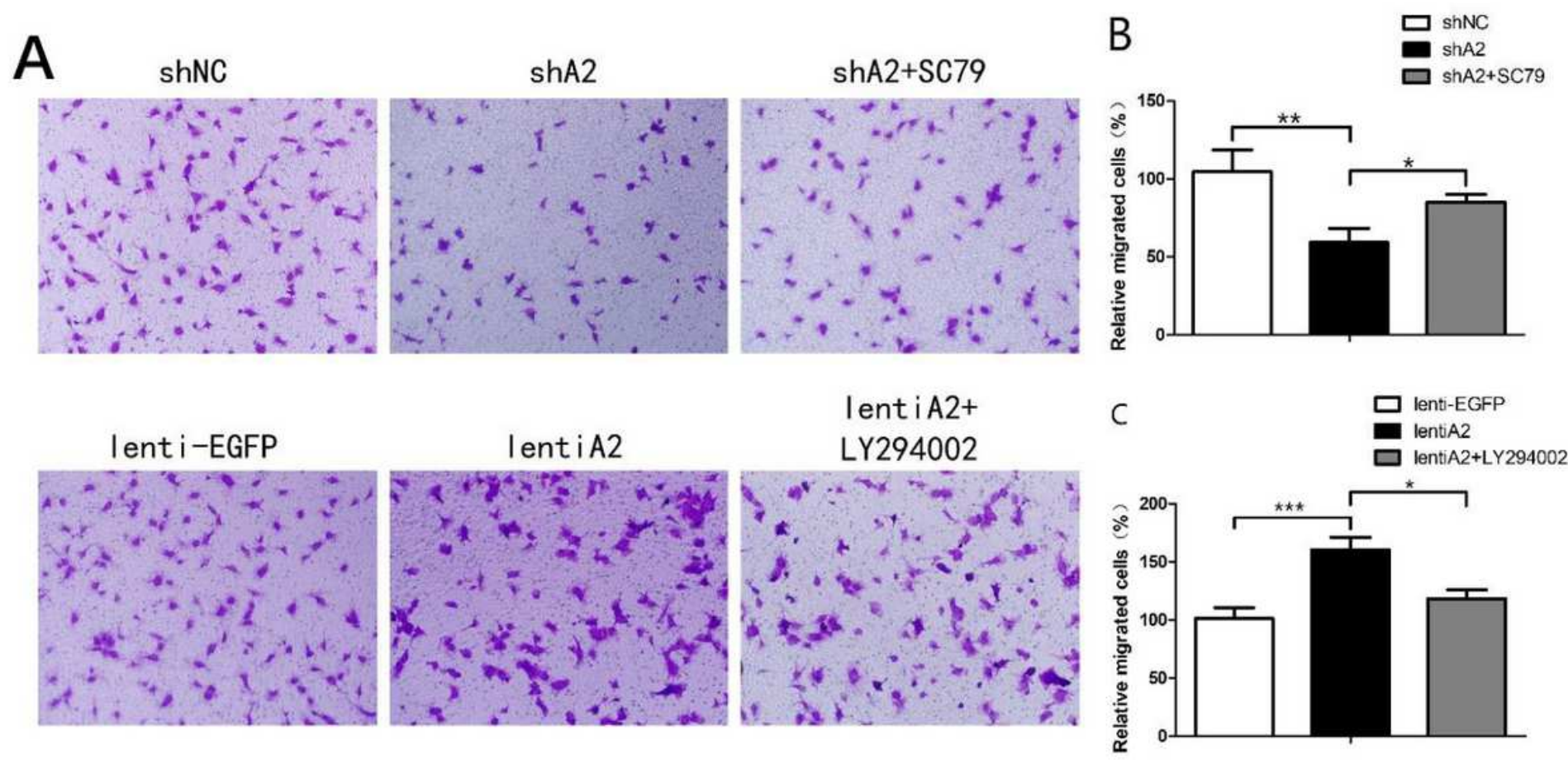

Figure 6

The interference and overexpression of ANXA2 obviously influence the migration and invasion of HRECs and the influence is highly related with PI3K/AKT signaling pathway. Transwell assay show the migration and invasion of HRECs in each group(A). Statistical analysis $(B, C) * P<0.05 \nabla^{* *} P<0.01 \rrbracket * * * P<0.001 \rrbracket n=3 \square$

A shNC

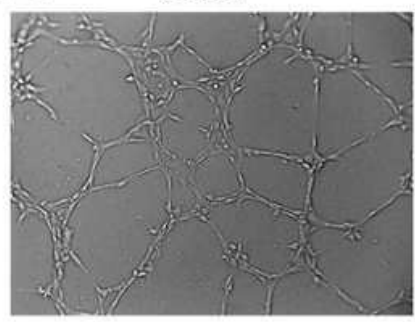

lenti-EGFP

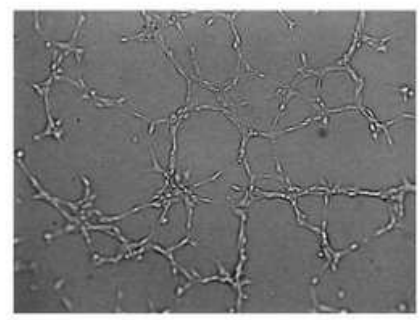

shA2

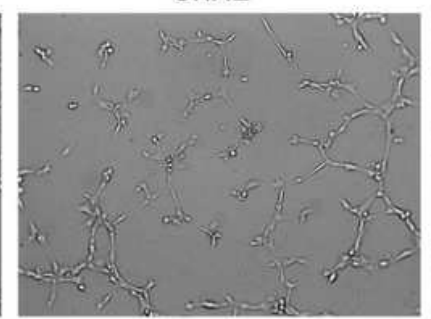

lentiA2

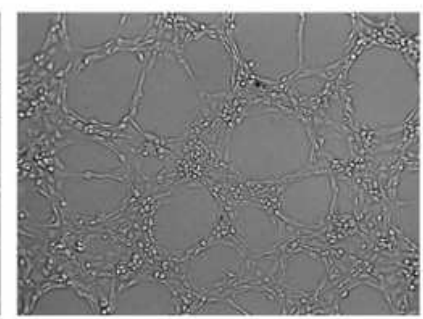

$\operatorname{shA2+SC79}$

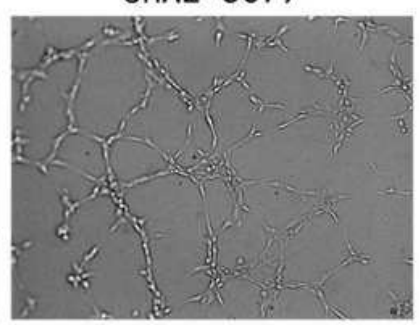

lentiA2+

LY294002

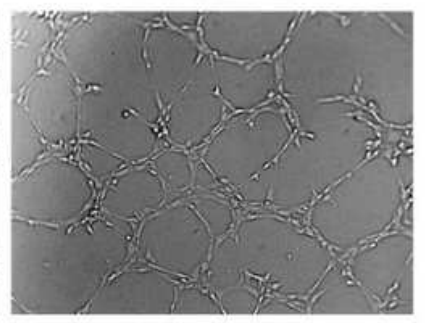

B 므 $\operatorname{snNC}$
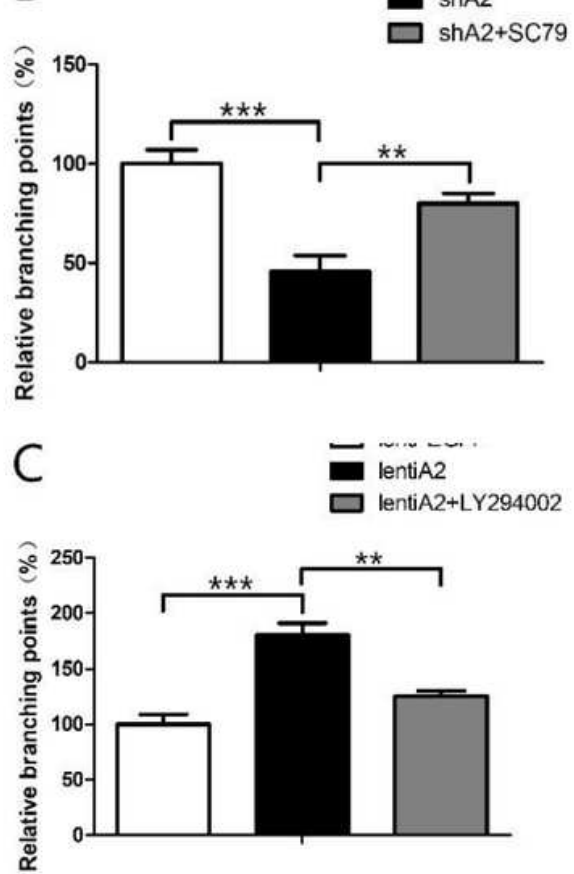

Figure 7 
The interference and overexpression of ANXA2 obviously influence the angiogenesis ability of HRECs and the influence is highly related with PI3K/AKT signaling pathway. Tube formation assay show the angiogenesis ability of HRECs in each group (A). Statistical analysis $(B, C){ }^{* *} P<0.01 \rrbracket * * * P<0.001 \rrbracket n=3 \square$

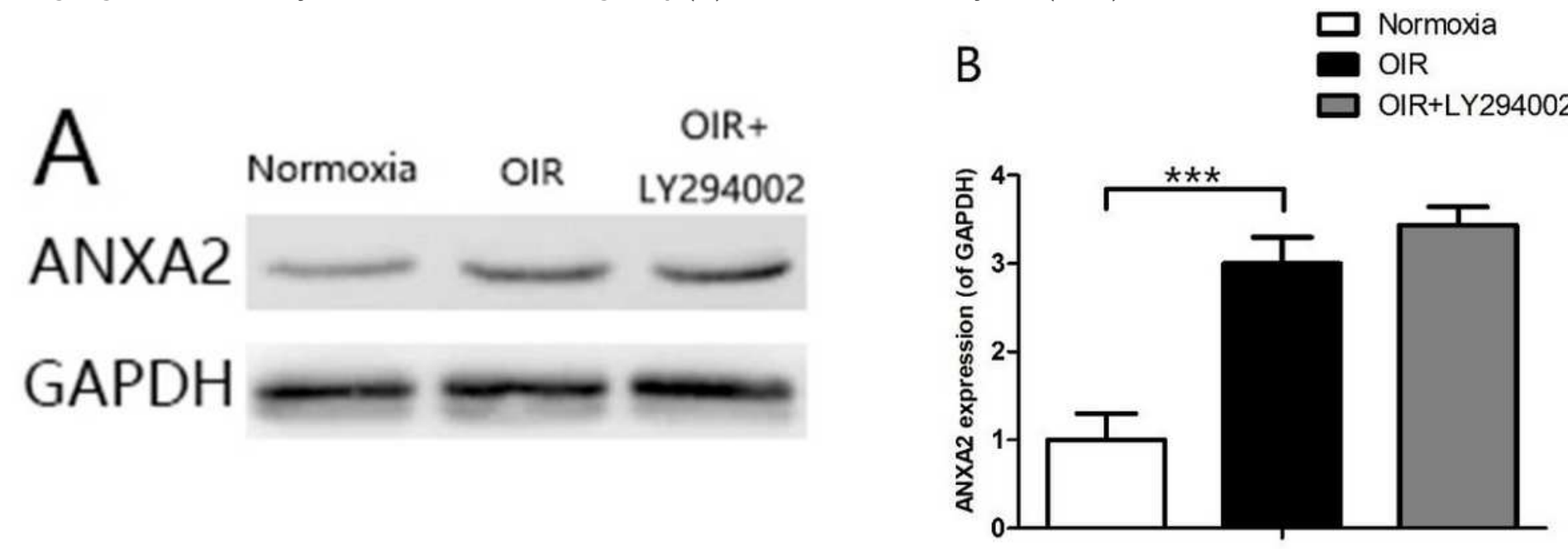

Figure 9

ANXA2 expression is significantly higher in retina of mice in OIR group and LY294002 treatment group. Western blot assay for protein expression of AXNA2 in retina of mice of each group (A); Statistical analysis $(B) * * * P<0.001 \rrbracket n=26$.
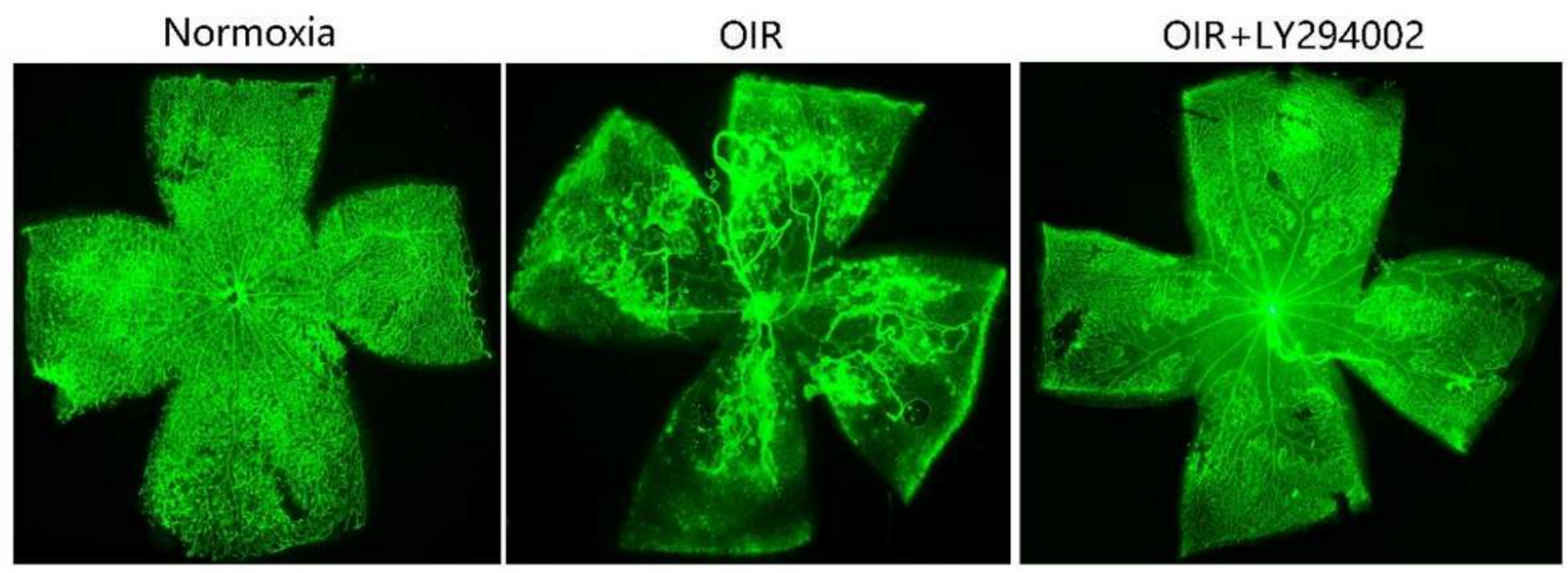

Figure 12

Inhibition of PI3K/AKT signaling pathway significantly recede the process of neovascularization. Images are typical retinal angiogram of the retina of mice in the normoxia group, OIR group and LY294002 treatment group. $\mathrm{n}=26$. 